Proceedings

\title{
Pyrrolidinodiones in Enol-Ugi, Enol-Passerini, and Anomalous Enol-Passerini Condensations ${ }^{\dagger}$
}

\author{
Ana G. Neo * and Carlos F. Marcos \\ Departamento de Química Orgánica e Inorgánica, Facultad de Veterinaria, Universidad de Extremadura. \\ Avda. Universidad, s/n, 10003 Cáceres, Spain; cfernan@unex.es \\ * Correspondence: aneo@unex.es; Tel.: +34-9-2725-7158; Fax: +34-9-2725-7110 \\ + Presented at the 22nd International Electronic Conference on Synthetic Organic Chemistry, 15 November- \\ 15 December 2018; Available Online: https://sciforum.net/conference/ecsoc-22.
}

Published: 19 November 2018

\begin{abstract}
In continuation of our recent research on the development of novel multicomponent reactions with isocyanides, we have used, for the first time, enols as the acid components in Ugiand Passerini-type reactions. Thus, electron-poor pyrrolidinodiones react with aldehydes, amines, and isocyanides to give the enaminic four-component adducts. Conversely, in the absence of the amine component, careful control of the reaction conditions allows the involvement of one or two molecules of isocyanide to afford, selectively, either Passerini-type or pseudo-enol-Ugi-type products. These unprecedented condensations of isocyanides, aldehydes, amines, and 4-substituted pyrrolidine-2,3-diones constitute an excellent strategy for the preparation of new biologically relevant pyrrolidinones having peptidic or pseudo-peptidic groups on carbon 3.
\end{abstract}

Keywords: multicomponent reactions; isocyanides; enamines; heterocycles.

\section{Introduction}

Pyrroles are important heterocycles that are present in many biomolecules, such as haem, chlorophyll, vitamin $\mathrm{B}_{12}$, and the bile pigments. Nonaromatic pyrrolidinones are also found in the structure of many natural products and biologically active molecules, such as inhibitors of cAMP phosphodiesterase [1], the vascular endothelial growth factor receptor (VEGF-R) [2], HIV integrase [3], and xanthine dehydrogenase (Figure 1) [4]. Importantly, structure-based design has recently identified 1,5-dihydropyrrol-2-ones containing oxygen and nitrogen substituents in position 3 as potent inhibitors of p53-MDM2 protein-protein interactions, which proved selectively active against tumor cells with deleted p53 and tumor xenograft models [5]. Although 3-substitued pyrrolidinones are readily accessible by the nucleophilic substitution on 2,3-diones [6-9], rather harsh conditions are required and only simple oxygen and nitrogen groups can be introduced $[4,5,10]$. Thus, it is crucial to find mild and selective procedures to introduce complex substituents on position 3 of pyrrolidinones. 
<smiles>[R]C1=C(c2ccc(-n3ccnc3[R])cc2)CNC1=O</smiles>

CAMP phosphodiesterase inhibitor<smiles>[R]c1cc(C2=C(O)C(=O)N([R])C2)ncn1</smiles>

HIV integrase inhibitor

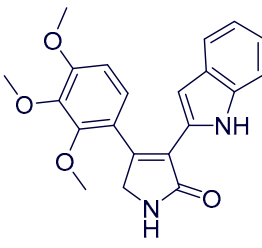

VEGF-R inhibitor

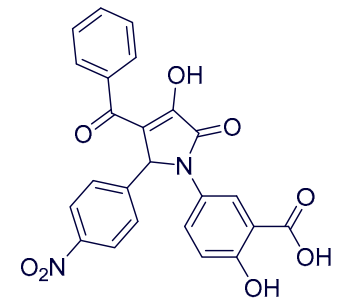

Stabilizer of PPIs interactions

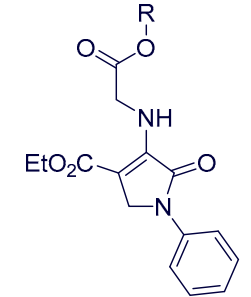

xanthine dehydrogenase inhibitor

Figure 1. Biologically relevant pyrrolidinones.

Multicomponent reactions (MCR) are highly convergent processes, in which three or more simple starting materials react to readily give complex products [11,12]. They are characterized by their great efficiency, versatility, and atom economy. Thus, MCR involving isocyanides (IMCRs), such as the Passerini three-component condensation (P3CC; Scheme 1) [13] and the Ugi four-component condensation (U4CC; Scheme 1) [14], are extensively used to synthesize diversely functionalized substituted $\alpha$-acyloxy- and $\alpha$-acylamino amides, respectively [15-17]. Further structural diversity can be achieved through a wide variety of postcondensation transformations [18-31] or by the replacement of one of the reactant components with a new reagent that mimics its reactivity and chemical behavior [12].

P3CC:

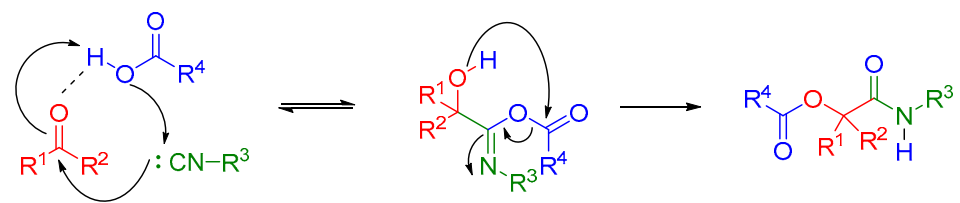

U4CC:

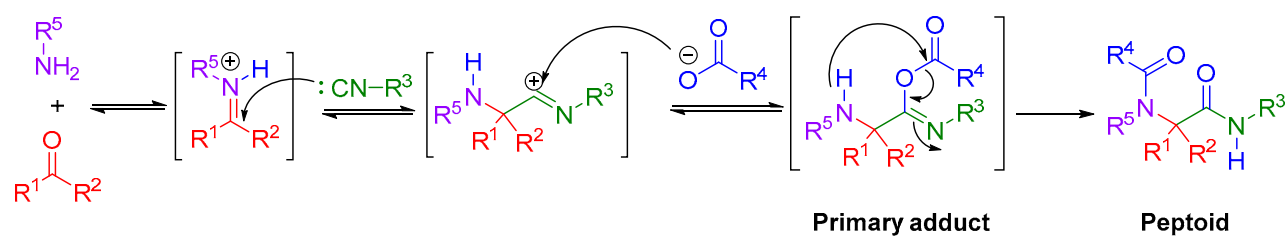

Scheme 1. Passerini and Ugi multicomponent condensations.

We have recently discovered that enols can be used as acid components in Passerini- and Ugi-type multicomponent condensations [32-35]. Here, we describe the use of enolic pyrrolidinodiones in different types of IMCRs leading to pyrrolidinone-derived enol-ethers and enamines.

\section{Results and Discussion}

Thus, the reaction of 4-hydroxy-5-oxo-2,5-dihydro-1H-pyrrole-3-carboxylates with isocyanides and either aldehydes or imines has been explored. 


\subsection{Enol-Ugi Condensations}

Enolic pyrrolidine-2,3-diones (1), imines (2), and isocyanides (3) were mixed in methanol at room temperature to give the expected Ugi-type adducts (4, Scheme 2$)$, which were isolated in very good to excellent yields. They were identified according to their spectral data, and the structure was further confirmed by X-ray diffraction analysis (Figure 2) [35].<smiles>[R1]C1C(=O)N(c2ccccc2)C(=O)C1O</smiles>

1

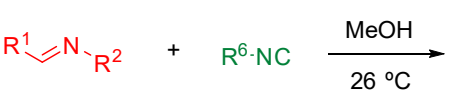

3

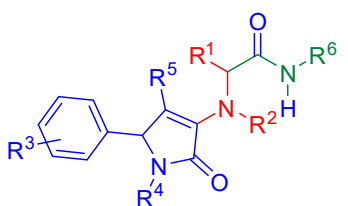

4

Scheme 2. Enol-Ugi condensation of pyrrolidinodiones.

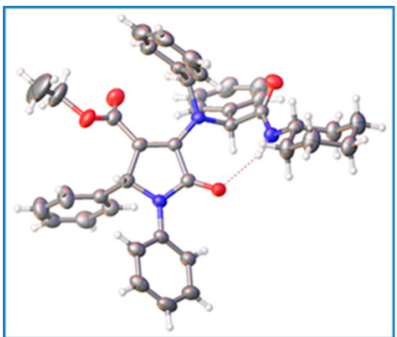

Figure 2. X-ray structure of the enol-Ugi adduct ethyl 4-(benzyl(2-(cyclohexylamino)-2-oxo-1phenylethyl)amino)-5-oxo-1,2-diphenyl-2,5-dihydro- $1 H$-pyrrole-3-carboxylate [35].

The reaction shows a wide scope and has been performed with different combinations of imines, isocyanides, and pyrrolidinodiones (Figure 3). We have also shown that it is possible to form the imines in situ from the corresponding amines and aldehydes; thus, the four-component condensation can be accomplished.

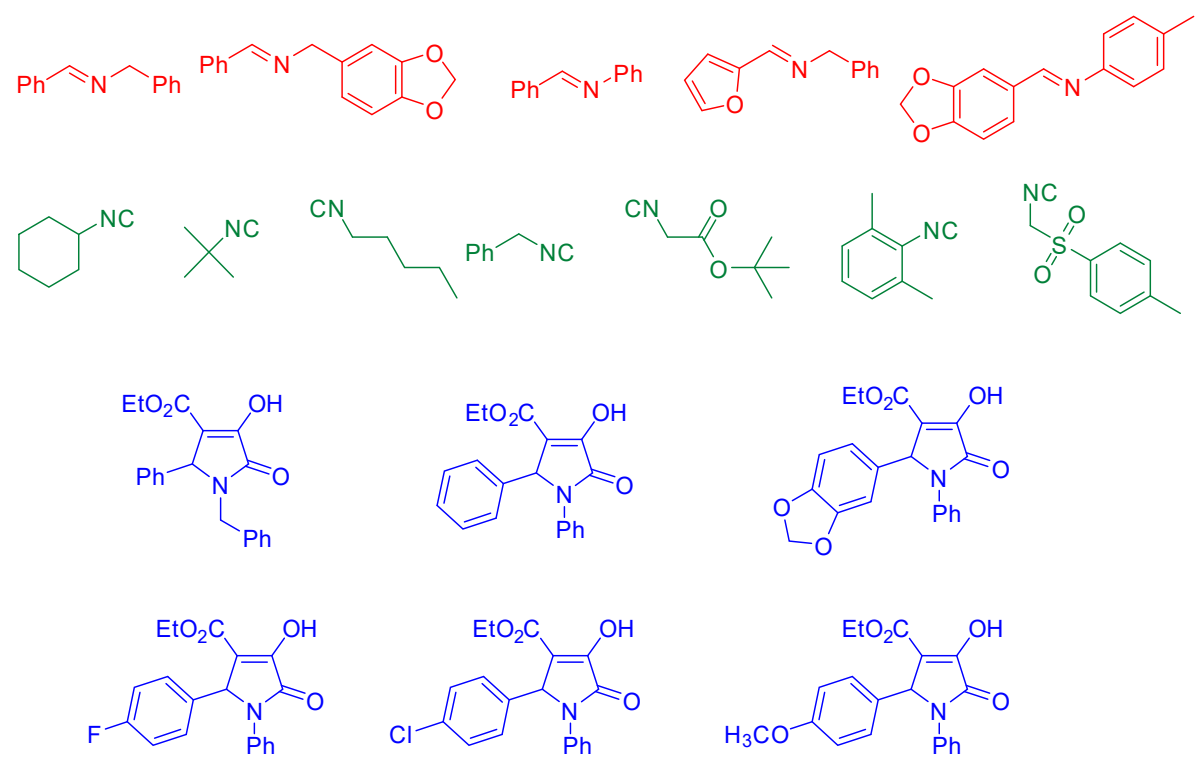

Figure 3. Scope of the enol-Ugi condensation with pyrrolidinodiones: different components used in the reaction.

The mechanism of the reaction can be explained by the protonation of the imine to form an iminium cation, which is attacked by the isocyanide to give a nitrilium cation intermediate. This then suffers the nucleophilic attack of the enolate, resulting in a primary adduct analogous to the primary adduct in the classical Ugi condensation. Thus, up to here, the reaction is mechanistically similar to 
the Ugi condensation with carboxylic acids. However, in the enol-Ugi condensation, the primary adducts spontaneously evolve to a stable product by a Michael-retro-Michael rearrangement, instead of the Mumm rearrangement that takes place in the classic U-4CC (Scheme 3).
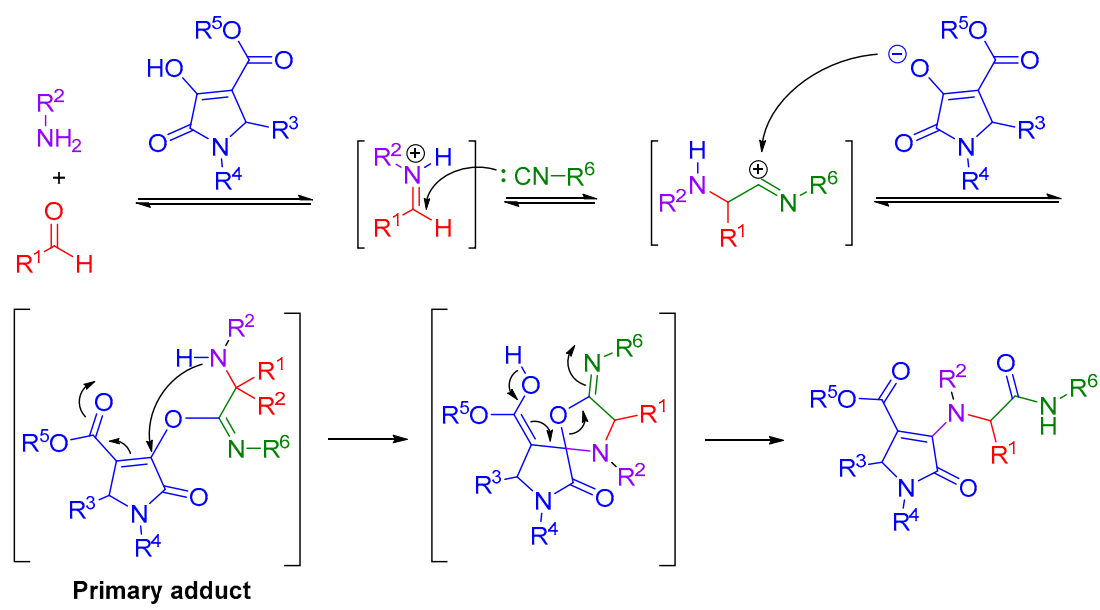

Scheme 3. Possible mechanism of the enol-Ugi condensation.

\subsection{Enol-Passerini Condensations}

In this case, the reaction was performed by mixing enolic pyrrolidine-2,3-diones (1), aldehydes (5), and isocyanides (3). The reaction takes place optimally at room temperature in dichloromethane, readily affording the corresponding pyrrolidinone amido ethers (6) in good to excellent yields [32]. The reaction is also possible in on-water conditions (i.e., using water as the solvent (Scheme 4)).

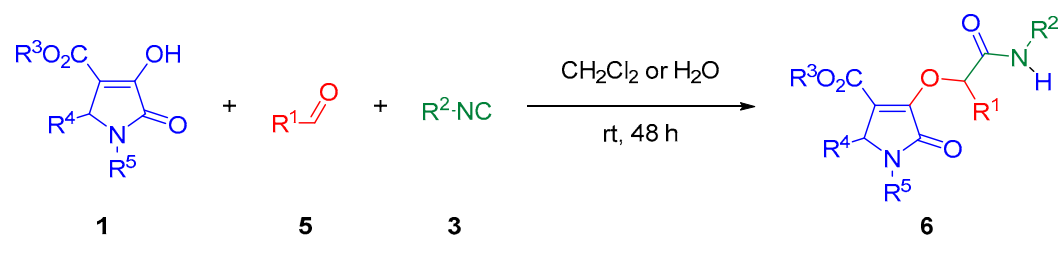

Scheme 4. Enol-Passerini condensation of pyrrolidinodiones.

The reaction has been also shown to be quite general, allowing the use of diverse aromatic aldehydes, pyrrolidinodiones, and both aliphatic and aromatic isocyanides (Figure 4).
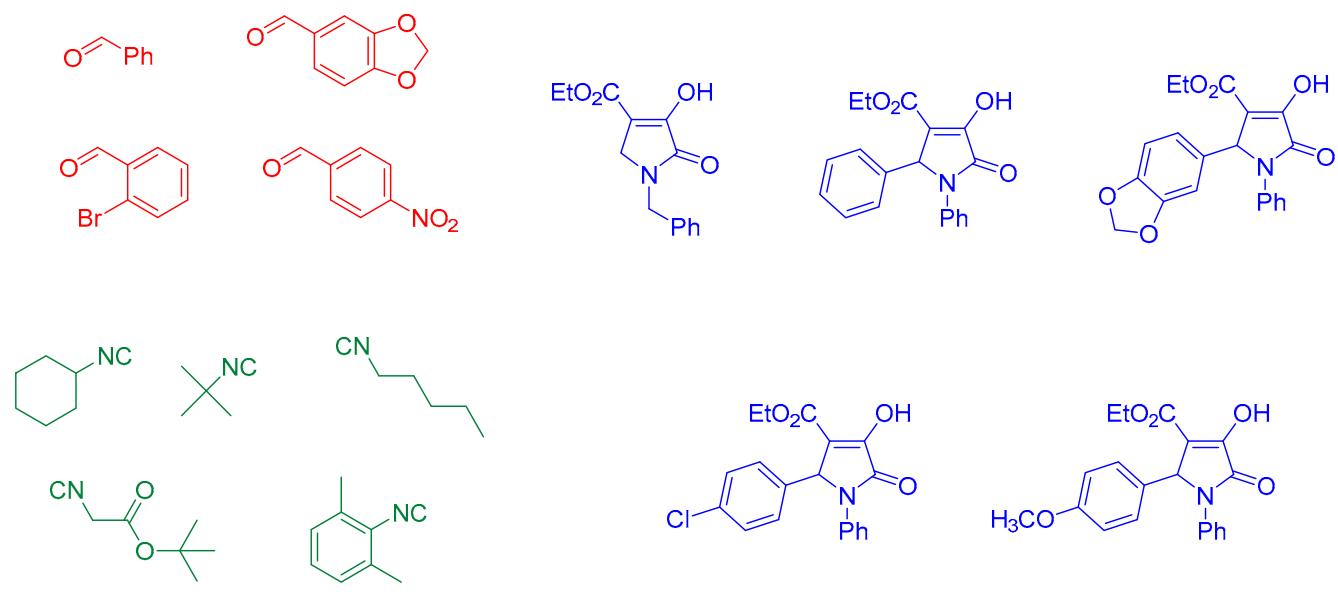

Figure 4. Scope of the enol-Passerini condensation with pyrrolidinodiones: different components used in the reaction. 
A mechanism in which the pyrrolidinodione acts as the acid component of the condensation can be envisaged. In parallel with the classical Passerini condensation, the reaction purportedly takes place in a concerted manner, as show in Scheme 5.

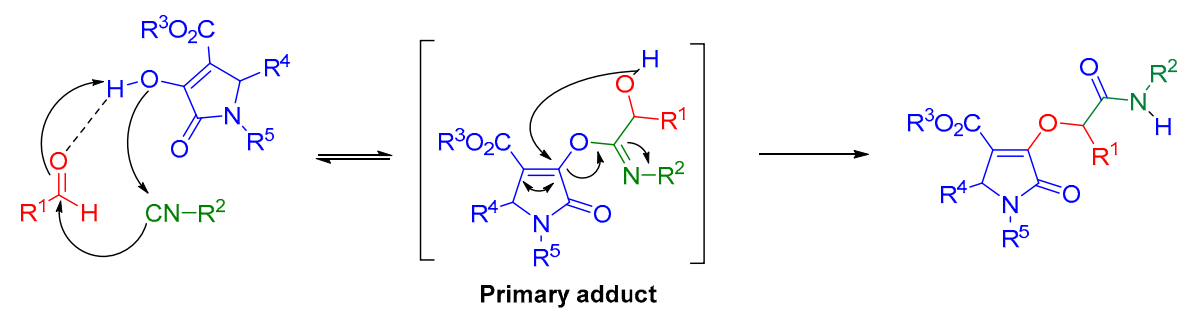

Scheme 5. Possible mechanism of the enol-Passerini condensation.

\subsection{Anomalous Enol-Passerini Condensations}

Finally, the reaction of pyrrolidine-2,3-diones (1), aldehydes (5), and two equivalents of isocyanides (3) in polar solvents, such as methanol, yielded a pseudo-enol-Ugi four-component adduct (7; Scheme 6) [32].

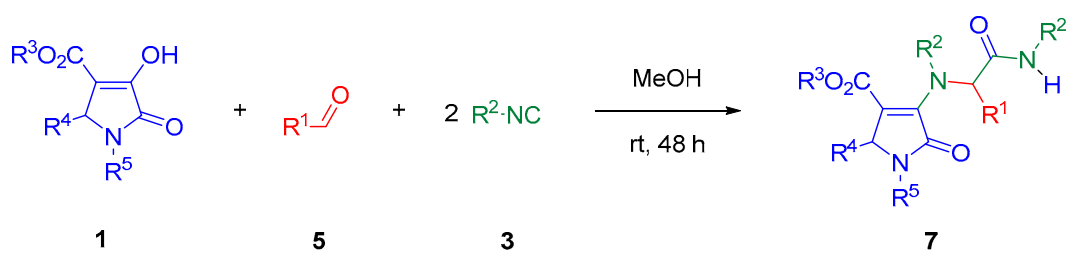

Scheme 6. Anomalous enol-Passerini condensation of pyrrolidinodiones.

In this case, the reaction admits diverse aldehydes and pyrrolidinodiones, but it is not possible with bulky isocyanides (Figure 5).

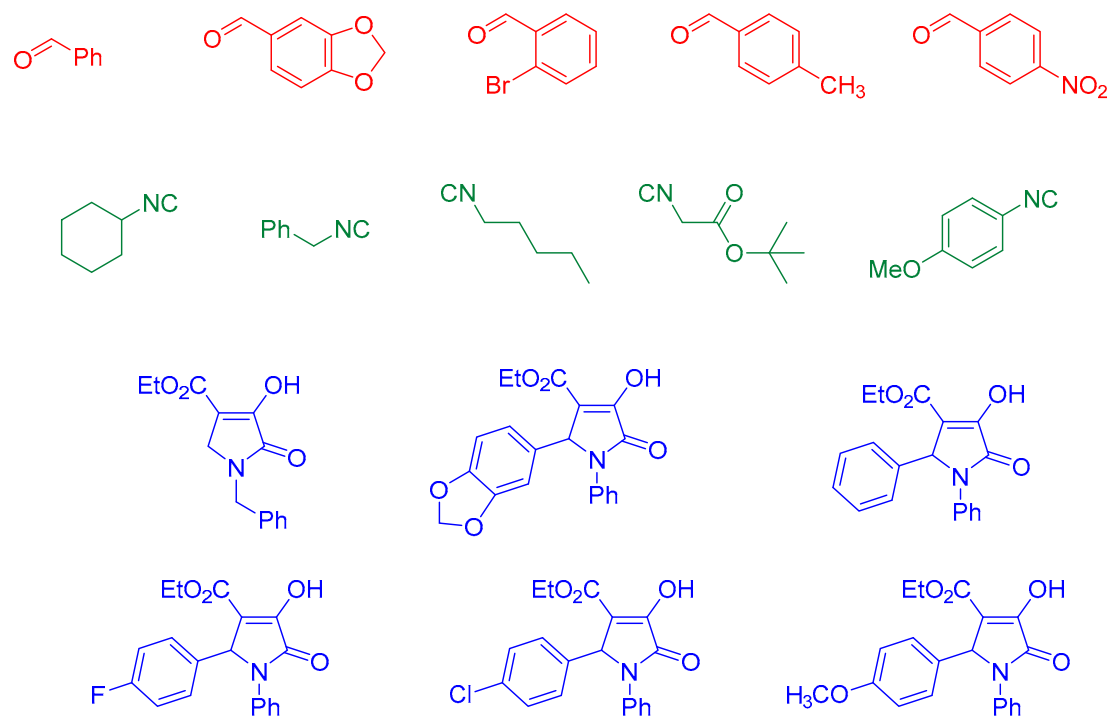

Figure 5. Scope of the anomalous enol-Passerini condensation with pyrrolidinodiones: different components used in the reaction.

The mechanism can be rationalized considering that a [2+2] cycloaddition of an aldehyde and a protonated isocyanide, followed by the cycloreversion and transfer of a formyl group to the solvent to give methyl formate and an intermediate iminium ion. This can then participate in a subsequent enol-Ugi reaction (Scheme 7). It had been previously observed that isocyanides and aldehydes can react to give imines in the presence of Brönsted [36] or Lewis acids [37]. 


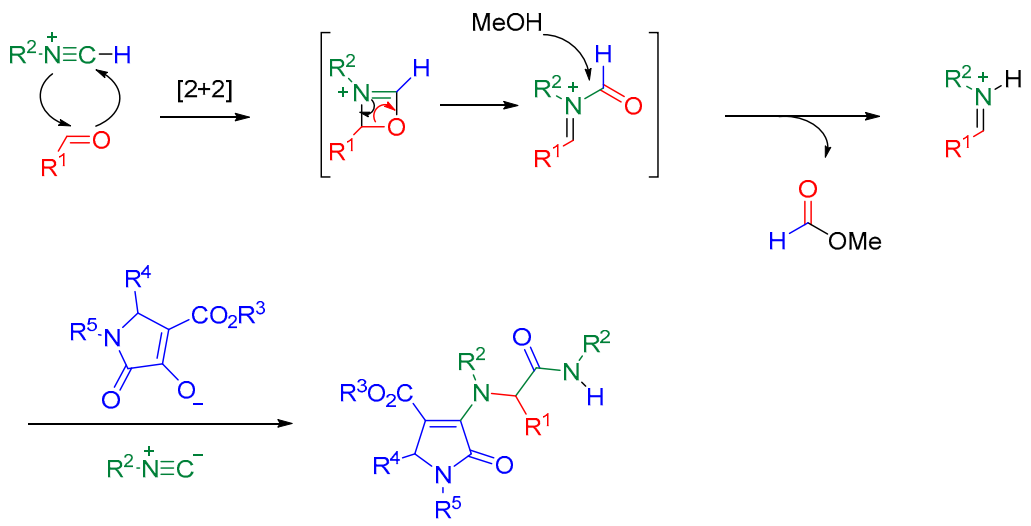

Scheme 7. Possible mechanism of the anomalous enol-Passerini condensation.

\section{Conclusions}

In summary, we have used enols for the first time as the acid component in Ugi- and Passerini-type reactions. Careful control of the reaction conditions allows us to obtain Ugi, Passerini, or pseudo-enol-Ugi products selectively. The use of enols as acidic partners in MCRI is an excellent strategy for the preparation of new pyrrolidinones having peptidic or pseudo-peptidic groups on carbon 3.

Acknowledgments:_We thank the financial support from Junta de Extremadura and FEDER (IB16095).

\section{References}

1. Lampe, J.W.; Chou, Y.L.; Hanna, R.G.; Di Meo, S.V.; Erhardt, P.W.; Hagedorn, A.A.; Ingebretsen, W.R.; Cantor, E. (imidazolylphenyl)pyrrol-2-one inhibitors of cardiac camp phosphodiesterase. J. Med. Chem. 1993, 36, 1041-1047, doi:10.1021/jm00060a012.

2. Peifer, C.; Selig, R.; Kinkel, K.; Ott, D.; Totzke, F.; Schächtele, C.; Heidenreich, R.; Röcken, M.; Schollmeyer, D.; Laufer, S. Design, synthesis, and biological evaluation of novel 3-aryl-4-(1h-indole-3yl)1,5-dihydro-2h-pyrrole-2-ones as vascular endothelial growth factor receptor (vegf-r) inhibitors. J. Med. Chem. 2008, 51, 3814-3824, doi:10.1021/jm8001185.

3. Kawasuji, T.; Fuji, M.; Yoshinaga, T.; Sato, A.; Fujiwara, T.; Kiyama, R. 3-hydroxy-1,5-dihydro-pyrrol-2-one derivatives as advanced inhibitors of hiv integrase. Biorg. Med. Chem. 2007, 15, 5487-5492, doi:10.1016/j.bmc.2007.05.052.

4. Jourdan, F.; Kaiser, J.T.; Lowe, D.J. Synthesis of new n-(5-oxo-2,5-dihydro)pyrrol-3-yl glycines and n-(5-oxo-2,5-dihydro)pyrrol-3-yl glycines esters. Synth. Commun. 2005, 35, 2453-2466, doi:10.1080/00397910500191219.

5. Zhuang, C.; Miao, Z.; Zhu, L.; Dong, G.; Guo, Z.; Wang, S.; Zhang, Y.; Wu, Y.; Yao, J.; Sheng, C.; et al. Discovery, synthesis, and biological evaluation of orally active pyrrolidone derivatives as novel inhibitors of p53-mdm2 protein-protein interaction. J. Med. Chem. 2012, 55, 9630-9642, doi:10.1021/jm300969t.

6. Wasserman, H.H.; Koch, R.C. Studies on 1,5-diphenyl-2,3-pyrrolidinedione and related compounds. J. Org. Chem. 1962, 27, 35-39, doi:10.1021/jo01048a008.

7. Metten, B.; Kostermans, M.; Van Baelen, G.; Smet, M.; Dehaen, W. Synthesis of 5-aryl-2-oxopyrrole derivatives as synthons for highly substituted pyrroles. Tetrahedron 2006, 62, 6018-6028, doi:10.1016/j.tet.2006.04.005.

8. Taylor, W.; Vadasz, A. The reactions of aromatic schiff bases with dimethyl acetylenedicarboxylate. Ii. Reaction in the presence of water. Aust. J. Chem. 1982, 35, 1227-1230, doi:10.1071/CH9821227.

9. Sun, J.; Wu, Q.; Xia, E.-Y.; Yan, C.-G. Molecular diversity of three-component reactions of aromatic aldehydes, arylamines, and acetylenedicarboxylates. Eur. J. Org. Chem. 2011, 2011, 2981-2986, doi:10.1002/ejoc.201100008.

10. Armisheva, M.; Kornienko, N.; Gein, V.; Vakhrin, M. Reactions of 5-aryl-4-acyl-1-(4-hydroxyphenyl)-3hydroxy-3-pyrrolin-2-ones with arylamines. Russ. J. Gen. Chem. 2011, 81, 1893, doi:10.1134/s107036321109026x. 
11. Domling, A.; Wang, W.; Wang, K. Chemistry and biology of multicomponent reactions. Chem. Rev. 2012, 112, 3083-3135, doi:10.1021/cr100233r.

12. Ganem, B. Strategies for innovation in multicomponent reaction design. ACC Chem. Res. 2009, 42, 463-472, doi:10.1021/ar800214s.

13. Passerini, M. Sopra gli isonitrili (1). Composto del p-isonitrilo-azobenzolo con acetone ed acido acetica. Gazz. Chim. Ital. 1921, 51(II), 126.

14. Ugi, I.; Meyr, R.; Fetzer, U.; Steinbrückner, C. Versuche mit isonitrilen. Angew. Chem. 1959, 71, 386.

15. Sadjadi, S.; Heravi, M.M.; Nazari, N. Isocyanide-based multicomponent reactions in the synthesis of heterocycles. RSC Adv. 2016, 6, 53203-53272, doi:10.1039/c6ra02143c.

16. Dömling, A. Recent developments in isocyanide based multicomponent reactions in applied chemistry. Chem. Rev. 2006, 106, 17-89, doi:10.1021/cr0505728.

17. Domling, A.; Ugi, I. Multicomponent reactions with isocyanides. Angew. Chem. Int. Ed. 2000, 39, 3169-3210, doi:10.1002/1521-3773(20000915)39:18<3168::AID-ANIE3168>3.0.CO;2-U.

18. Neo, A.G.; López-García, L.; Marcos, C.F. Allylic amination of passerini adducts. Application to the selective synthesis of chromone-substituted $\alpha$-and $\gamma$-amino acid peptidic and retropeptidic units. RSC Advances 2014, 4, 40044-40053, doi:10.1039/c4ra05719h.

19. Neo, A.; Carrillo, R.; Delgado, J.; Marcaccini, S.; Marcos, C.F. A multicomponent approach to the synthesis of 1,3-dicarbonylic compounds. Mol. Divers. 2011, 15, 529-539, doi:10.1007/s11030-010-9277-y.

20. Marcos, C.F.; Marcaccini, S.; Menchi, G.; Pepino, R.; Torroba, T. Studies on isocyanides: Synthesis of tetrazolyl-isoindolinones via tandem ugi four-component condensation/intramolecular amidation. Tetrahedron Lett. 2008, 49, 149-152, doi:10.1016/j.tetlet.2007.10.154.

21. Faggi, C.; Neo, A.G.; Marcaccini, S.; Menchi, G.; Revuelta, J. Ugi four-component condensation with two cleavable components: The easiest synthesis of 2,n-diarylglycines. Tetrahedron Lett. 2008, 49, 2099-2102, doi:10.1016/j.tetlet.2008.01.134.

22. Neo, A.G.; Carrillo, R.M.; Barriga, S.; Moman, E.; Marcaccini, S.; Marcos, C.F. Multicomponent synthesis of highly substituted 2-pyridones. Synlett 2007, 327-329, doi:10.1055/s-2007-967992.

23. Carrillo, R.M.; Neo, A.G.; Lopez-Garcia, L.; Marcaccini, S.; Marcos, C.F. Zinc catalysed ester solvolysis. Application to the synthesis of tartronic acid derivatives. Green Chem. 2006, 8, 787-789, doi:10.1039/b607258a.

24. Neo, A.G.; Marcos, C.F.; Marcaccini, S.; Pepino, R. Studies on isocyanides. A facile synthesis of 4,5-dihydro-1,4-benzothiazepin-3(2h)-ones via post-condensation modifications of the ugi reaction. Tetrahedron Lett. 2005, 46, 7977-7979, doi:10.1016/j.tetlet.2005.09.071.

25. Neo, A.G.; Delgado, J.; Polo, C.; Marcaccini, S.; Marcos, C.F. A new synthesis of beta-keto amides by reduction of passerini adducts. Tetrahedron Lett. 2005, 46, 23-26, doi:10.1016/j.tetlet.2004.11.041.

26. Marcos, C.F.; Marcaccini, S.; Pepino, R.; Polo, C.; Torroba, T. Studies on isocyanides and related compounds; a facile synthesis of functionalized 3(2h)-pyridazinones via ugi four-component condensation. Synthesis 2003, 691-694, doi:10.1055/s-2003-38071.

27. Marcaccini, S.; Pepino, R.; Marcos, C.F.; Polo, C.; Torroba, T. Studies on isocyanides and related compounds. Synthesis of furan derivatives and their transformation into indole derivatives. J. Heterocycl. Chem. 2000, 37, 1501-1503, doi:10.1002/jhet.5570370615.

28. Bossio, R.; Marcaccini, S.; Pepino, R.; Marcos, C.F. Multicomponent reactions-A convenient undergraduate organic chemistry experiment. J. Chem. Educ. 2000, 77, 382, doi:10.1021/ed077p382.

29. Bossio, R.; Marcos, C.F.; Marcaccini, S.; Pepino, R. Studies on isocyanides and related compounds. An unusual synthesis of functionalized succinimides. Synthesis 1997, 1389-1390, doi:10.1055/s-1997-1367 .

30. Bossio, R.; Marcos, C.F.; Marcaccini, S.; Pepino, R. Studies on isocyanides and related compounds. A novel synthetic route to 1,6-dihydro-6-oxopyridine-2-carboxylic acid derivatives. Heterocycles 1997, 45, 1589 1592, doi:10.3987/COM-97-7855.

31. Bossio, R.; Marcos, C.F.; Marcaccini, S.; Pepino, R. A facile synthesis of beta-lactams based on the isocyanide chemistry. Tetrahedron Lett. 1997, 38, 2519-2520, doi:10.1016/s0040-4039(97)00389-4.

32. Neo, A.G.; Marcos, C.F. Selective synthesis of 3-substituted pyrrolidinones by enol-passerini and anomalous enol-passerini condensations. Org. Lett. 2018, 20, 3875-3878, doi:10.1021/acs.orglett.8b01462.

33. Neo, A.G.; Castellano, T.G.; Marcos, C.F. An easy synthesis of diversely functionalized 2h-chromenes and amido amines by an enol-ugi reaction. Arkivoc 2017, 2017, 21-31, doi:10.3998/ark.5550190.p009.775. 
34. Neo, A.G.; Castellano, T.G.; Marcos, C.F. Enol-ugi reaction of hydroxycoumarins: Straightforward synthesis of amino acid derived coumarin enamines. Synthesis 2015, 47, 2431-2438, doi:10.1055/s-0034-1380436.

35. Castellano, T.G.; Neo, A.G.; Marcaccini, S.; Marcos, C.F. Enols as feasible acid components in the ugi condensation. Org. Lett. 2012, 14, 6218-6221, doi:10.1021/ol302976g.

36. Okandeji, B.O.; Sello, J.K. Brønsted acidity of substrates influences the outcome of passerini three-component reactions. J. Org. Chem. 2009, 74, 5067-5070, doi:10.1021/jo900831n.

37. Dai, W.-M.; Li, H. Lewis acid-catalyzed formation of ugi four-component reaction product from passerini three-component reaction system without an added amine. Tetrahedron 2007, 63, 12866-12876, doi:10.1016/j.tet.2007.10.050.

(C) 2019 by the authors. Licensee MDPI, Basel, Switzerland. This article is an open access article distributed under the terms and conditions of the Creative Commons Attribution (CC BY) license (http://creativecommons.org/licenses/by/4.0/). 